\title{
DIFFRACTIVE LASER OPTICAL ENCODER WITH HIGH TOLERANCE TO HIGH-SPEED MECHANICAL RUNOUT
}

\author{
Chyan-Chyi $\mathrm{Wu}^{1}$, Chih-Kung Lee ${ }^{2 *}$, Shui-Shong Lu ${ }^{1}$, Wen-Jong Chen ${ }^{1}$, Ching-Sang Yang ${ }^{2}$, \\ and Chi-Tang $\mathrm{Hsieh}^{3}$ \\ ${ }^{1}$ Department of Mechanical Engineering, \\ National Taiwan University, \\ Taipei, Taiwan 106, R.O.C. \\ ${ }^{2}$ Institute of Applied Mechanics, \\ National Taiwan University, \\ Taipei, Taiwan 106, R.O.C. \\ ${ }^{3}$ AHEAD Optoelectronics, Inc. \\ Taipei, Taiwan 235, R.O.C.
}

Key Words: high-speed mechanical runout, error analysis, 1-x telescope, conical diffraction.

\begin{abstract}
This paper is concerned with the design of a laser linear encoder with high head-to-scale alignment tolerance. It adopts $1-x$ telescope design that can increase by 6 to 20 times the head-to-scale tolerance of existing encoders of the same class. The optical design of the laser encoder can avoid effects from differences in polarization diffraction efficiencies. In other designs the non-uniformity of the temperature field within the head-to-scale range yields a nonzero initial phase and thus decreases the measurement accuracy of the system. The misalignment of the polarizers for the circular polarization interferometer configuration makes for both inclined and elliptical quadrature signals in the output. For an encoder system which adopts an arctangent algorithm, this effect may lower the measuring resolution. The resolution of our laser encoder design is verified by experiments.
\end{abstract}

\section{INTRODUCTION}

Laser encoders, which provide accurate linear and angular displacement information with resolution down to the nanometer range, are commonly used in today's high-tech applications. Many state-of-theart linear laser encoders exist today. For example, Canon made a compact laser optical encoder by utilizing the cat's eye design (Nishimura et al., 1990a; Nishimura et al., 1990b). In Europe, Heidenhain proposed a diffraction encoder of the integrated optical type (Olivier and Francois, 1990). Besides, IBM proposed a wavefront reconstruction optics so as to use the laser encoder as an actuator position sensor (Chiang and Lee, 1995). Mitutoyo proposed a linear encoder with improved detection signal insensitivity to optical grating gap variation (Souji, Hideki and Seiji, 1990) and corrected the adverse polarization effect on the diffracted beams induced by the grating scale (Masreleiz, 1992).

The main bottleneck in making wide applications of the laser encoders lies in the fact that the optical head of a laser encoder must not only be aligned with micrometer precision but also be held

*Correspondence addressee 


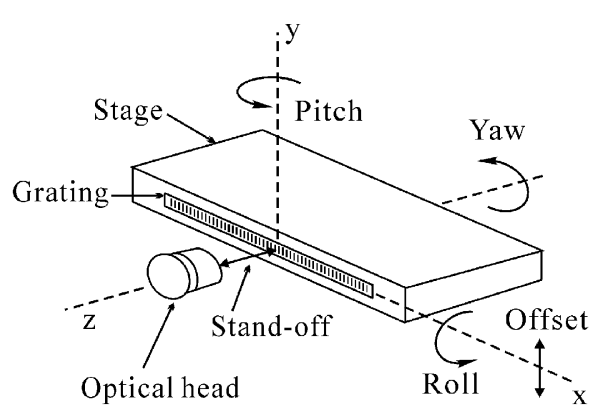

Fig. 1 Schematics of the mechanical runouts that may influence the performance of the encoders. The runouts are defined from the optical head to the stage and thus may be different from traditional stage runout definitions

to tight tolerances during system operation. The highspeed operation capacity of production machines is very important for current high-tech industries such as semiconductors, DVDs and hard disks. Typically, higher operating speeds of the machines corresponds to worse mechanical runouts. As shown in Fig. 1, there exist five axes of mechanical runout between the optical head and the operating machine. The mechanical runout of the specimen, i.e., the machine, where the grating scale is mounted, will directly influence the optical signals and may cause the optoelectronic signals to decay or even to disappear. In addition, information decoded may lead to erroneous measurement results when the runout is present. The IBM laser optical encoder design, which was proposed to tackle the high-speed mechanical runout problem the Canon encoder encounters, is shown in Fig. 2. The main difference between the optical design of these two encoders is that the Canon encoder adopts a cat's eye reflector and the proposed encoder design derived from improving the IBM laser encoder utilizes a design termed $1-x$ telescope. The cat's eye reflector is made by coating only the center portion of the back plane of a 1/4 pitch GRIN lens, which has the advantage of being small in size and easy to align. On the other hand, the $1-x$ telescope design concept shown in Fig. 2 is achieved by placing the front focal plane of the wavefront reconstruction lens at the midpoint location where the incident and return light beams impinge on the reflective grating. In addition, the mirrors in Fig. 2 are placed at the back focal plane of the wavefront reconstruction lens. Due to the geometry of the GRIN lens, the cat's eye reflector can never be aligned to have the $1-x$ telescope configuration unless the front surface of the GRIN lens is placed in intimate contact with the reflective grating.

Principles of the optical system design are described in detail herein by describing the optical paths in detail first. Then the error analysis of the head-toscale mechanical runout will be given. Experimental

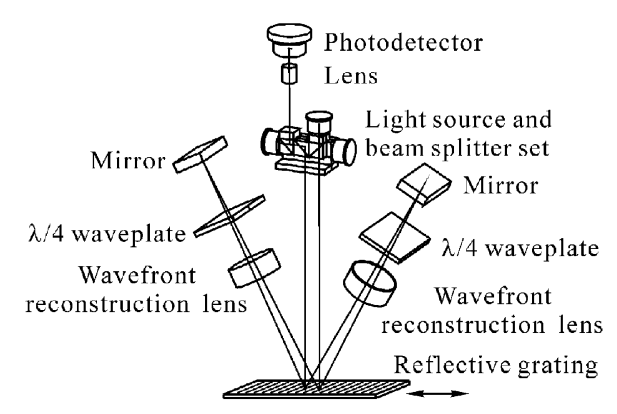

Fig. 2 Schematic of the IBM laser linear encoder

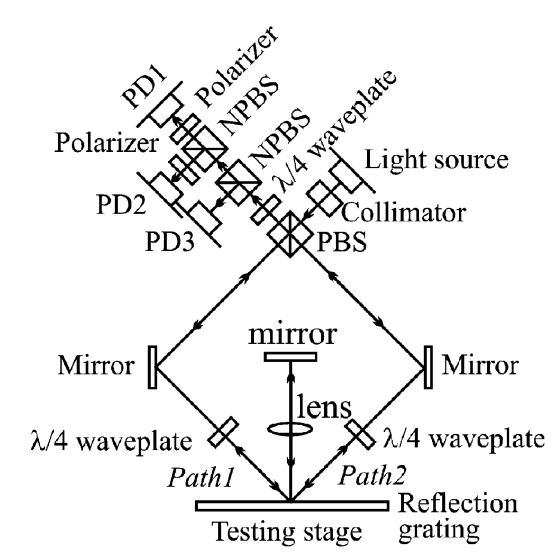

Fig. 3 Schematics of the newly proposed linear encoder

results obtained by comparing the signal output obtained from the newly proposed laser encoder with that of an $\mathrm{HP}^{\mathrm{TM}}$ laser interferometer are to be examined as well.

\section{THEORY OF LASER ENCODER}

In Fig. 3, a linearly polarized light emitted from a diode laser is collimated and aligned to make sure that the two light beams split by the polarization beam splitter (PBS) will have equal intensity. After $p$-wave (TM wave) transmitting through the PBS and traveling along the left half of the optical path, the quarter waveplate converts the $p$-wave into a circularly polarized light beam. Similarly the $s$-wave (TE wave) traveling along the right half of the optical path is converted into a circularly polarized light beam as well. These two orthogonal circularly polarized light beams diffracted by the reflective grating twice are then encoded with the displacement information of the specimen on which the grating is attached.

The $p$ - and $s$ - polarized light beams are recombined at the PBS. The optical configuration shown in Fig. 3 will make sure that the polarization states of these two light beams are orthogonal to each other. These two orthogonally polarized light beams will become right and left circularly polarized after passing the quarter waveplate. The combination of these 
two circularly polarized light beams forms effective linearly polarized light beams whose azimuths are functions of the grating displacement. These two polarized light beams produce the quadrature signals from the photodetectors, then the phase decoding algorithm is employed for the signal processing (Lee et al., 1999; Wu et al., 1999). More specifically, the polarizing plates located in front of the photodetectors PD1 and PD2 are arranged to have a $45^{\circ}$ angle with respect to each other within the polarization state space. The light intensities measured by PD1 and PD2, forming the quadrature signals, $I_{1}$ and $I_{2}$, respectively are given by the following equation

$$
\begin{aligned}
& I_{1}=D C_{1}+A_{1} \cos (\eta), \\
& I_{2}=D C_{2}+A_{2} \sin (\eta-\delta),
\end{aligned}
$$

where $D C_{1}$ and $D C_{2}$ are the amplitudes of the DC components, $A_{1}$ and $A_{2}$ the amplitudes of the AC components, $\eta$ is the relative phase difference of the two beams due to the displacement of the grating scale, and $\delta$ the additional phase difference between the two photo detectors receiving the $I_{1}, I_{2}$ signals. A grating scale displacement of $\Delta x$ causes a double Doppler shift (Drain, 1980; Durst and Whitelaw, 1976) which is related to the phase difference $\eta$ for a first order diffraction by the following equation

$$
\eta=8 \pi \Delta x / d,
$$

where $d$ is the grating pitch. The phase difference between these two beams depends on the position of the diffraction grating. As the diffraction grating moves, the phase relationships of these two beams change, causing them to constructively or destructively interfere. By Eq. (3), the peak-to-peak period of the interfering beams within the encoder is $d / 4$. By interpolation of the quadrature signal electronically, for example, the measuring resolution of the laser encoder system can be improved to better than $0.1 \mathrm{~nm}$ with a grating pitch of $1.6 \mu \mathrm{m}$ and 4096 digitization resolution.

\section{1- $X$ TELESCOPE DESIGN}

The $1-x$ telescope design adopted in this optical head can be described by using the matrix optics approach as follows (Guenther, 1990):

$$
\begin{aligned}
& {\left[\begin{array}{cc}
1 & -f \\
0 & 1
\end{array}\right]\left[\begin{array}{cc}
1 & 0 \\
1 / f & 1
\end{array}\right]\left[\begin{array}{cc}
1 & -f \\
0 & 1
\end{array}\right]\left[\begin{array}{cc}
1 & 0 \\
0 & -1
\end{array}\right]\left[\begin{array}{cc}
1 & f \\
0 & 1
\end{array}\right]\left[\begin{array}{cc}
1 & 0 \\
-1 / f & 1
\end{array}\right]} \\
& \cdot\left[\begin{array}{ll}
1 & f \\
0 & 1
\end{array}\right]\left\{\begin{array}{c}
h_{0} \\
\alpha_{0}
\end{array}\right\}=\left\{\begin{array}{c}
-h_{0} \\
\alpha_{0}
\end{array}\right\}
\end{aligned}
$$

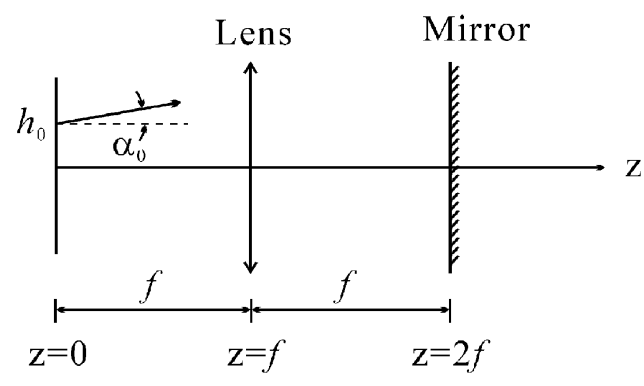

Fig. 4 Schematics of the $1-x$ telescope

where $\left(h_{0}, \alpha_{0}\right)$ represents the corresponding position and direction angles of the diffracted beam from the moving grating scale surface, and $f$ is the focal length of the lens as shown in Fig. 4. By Eq. (4), it can be shown that the output beam, transferred by the $1-x$ telescope optical system, traces along the opposite direction to the diffracted beam, and its output position located at $-h_{0}$ is symmetric to the optical axis of the 1-x telescope optical system with the position of the diffracted beam. Thus three advantages for the $1-x$ telescope can be clearly identified by using the results shown in Eq. (4). First, it assures that a second time diffracted beam can trace along an optical path that is parallel to the original one. Second, it provides the returned light beams with offsets avoiding the returned beams going back into the laser cavity to de-stabilize the laser. Third, even for the defocused case, which shows up under an additional spacing between the optical head and the grating scale, the output direction of the returned beam can still be held on an opposite line that is parallel to its original one. Therefore, this optical design makes the newly developed encoder system more robust than others.

\section{ERROR ANALYSIS}

The $I_{1}$ and $I_{2}$ signals trace out a trajectory in the intensity space. The position and shape of the signal trajectory depend on the $D C_{1}, D C_{2}, A_{1}$ and $A_{2}$ levels and thus influence the output accuracy of the signal processing using the arctangent algorithm. The headto-scale and photodetector module alignment errors will be detailed herein.

\section{Effect of Differences in Polarization Diffraction Efficiency}

For past laser encoders, errors stemming from the different diffraction efficiencies for incident light beams of different polarization states are one of the main design issues (Hutley, 1982; Masreliez, 1992). Although Mitutoyo's design claims that it can 
correct the effect of differences of polarization diffraction efficiency (Masreliez, 1992), it adopts an improper physical concept that the efficiencies of the grating diffraction have reversibility and thus may cause a system measurement error. In comparison, as the proposed laser encoder adopts a circular polarization interferometer configuration and thus is independent of polarization differences of diffraction efficiency, the quadrature signals are of the same amplitude. It can be shown clearly that incident light beams after two diffractions from the grating scale (Fig. 3) lead to a resultant electric field $E$ located in front of the second NPB that consists of left-circularly and a right-circularly polarized components with the same amplitude (see Appendix 1 for proof). The corresponding quadrature signal can be shown as follows

$$
\begin{aligned}
& I_{1}=D C+A C \cdot \cos (4 \cdot \Delta \omega \cdot t), \\
& I_{2}=D C+A C \cdot \sin (4 \cdot \Delta \omega \cdot t),
\end{aligned}
$$

where $D C, A C$ are the amplitudes of the $D C, A C$ components of the quadrature signal respectively, $t$ the time interval of the grating scale movement and

$$
A C=D C=G\left[k_{p}(\theta) k_{p}(0)+k_{s}(\theta) k_{s}(0)\right]^{2},
$$

where $G$ is a proportional constant, $\Delta \omega$ is the Doppler angular frequency shift and the $k_{p}(\theta), k_{p}(0)$, $k_{s}(\theta)$ and $k_{s}(0)$ are the first order diffraction efficiencies with respect to the $\theta$ and 0 incident angles for $p$ - and $s$-polarizations respectively. From Eqs. (5)(7), it is clear that the optical design of the newly developed laser encoder does not distort the output signal and the output resolution of the phase decoding is free from the effect of differences in the diffraction efficiencies for incident light beams with different polarization states. That is, the laser encoder remains in optimum operating condition even for cases where the difference in diffraction efficiencies exists for incident light with different polarization states.

\section{Effect of Temperature}

It is very difficult to keep this system temperature completely balanced; i.e. optical path differences (OPD) do exist between path 1 and path2, which are optical paths respectively along the left and right optical paths. Temperature variation influences the measurement accuracy with an additional phase $\gamma$ for the phase decoding and it can be shown easily by using Jones calculus as follows

$$
\eta=4 \cdot \Delta \omega \cdot t+\gamma
$$

where $t$ is the time interval during the grating scale movement, $\gamma$, the temperature variation induced phase error can be represented as follows

$$
\gamma=\frac{4 \pi}{\lambda}\left[\int_{p a t h 1} \Delta n \cdot d l-\int_{p a t h 2} \Delta n \cdot d l\right],
$$

where $\lambda$ is the wavelength of the laser source, $\Delta n$, is the index change induced by the temperature variation within the optical system with respect to the standard ambient temperature $20^{\circ} \mathrm{C}, d l$ is the infinitesimal path along path 1 or path 2 and

$$
\Delta n=n(x, z)-n_{0},
$$

where $n(x, z)$ is the index distribution caused by the temperature variation, and $n_{0}$ is the reference index at $20^{\circ} \mathrm{C}$ constant ambient temperature. Out of the laser encoder, only the head-to-scale region is exposed directly to the ambient environment and thus we can reasonably assume that the region within the optical head has a uniform temperature field. For example, consider a head-to-scale region of $1 \mathrm{~mm}$ spacing, path 1 with a uniform temperature variation, path2 with the same temperature as the reference temperature, $20^{\circ} \mathrm{C}$, which is the ambient temperature and a laser source with a wavelength at $633 \mathrm{~nm}$. By Eqs. (8-10) and the empirical refractive index formula, which is a function of temperature variation (Smith, 1990), we can estimate an error sensitivity of the displacement measurement, $1.1721 \mathrm{~nm} /{ }^{\circ} \mathrm{C}$ as shown in Fig. 5. Thus the temperature variation is radically significant for the measurement accuracy of the laser encoder system.

\section{The Polarization Misalignment of the Detector Modules}

It should be noted that errors do occur when the two PBSs in front of the corresponding photodetectors are misaligned at wrong relative polarization angles. By Jones calculus the misalignment angle $\xi$ can be related to the polarization misalignment error which shows up as an additional phase difference $\delta$ in the quadrature signals in Eq. (2), and

$$
\delta=2 \cdot \xi \text {. }
$$

(see Appendix 2 for proof). This is true especially because the polarization misalignment $\varepsilon(\eta)$ at detector modules induced error is a periodic error with respect to the phase $\eta$ of the quadrature signal. As the arctangent algorithm is applied, the error $\varepsilon(\eta)$ can be easily written down as follows

$$
\varepsilon(\eta)=\tan ^{-1}\left[\frac{\sin (\eta-\delta)}{\cos \eta}\right]-\eta,
$$




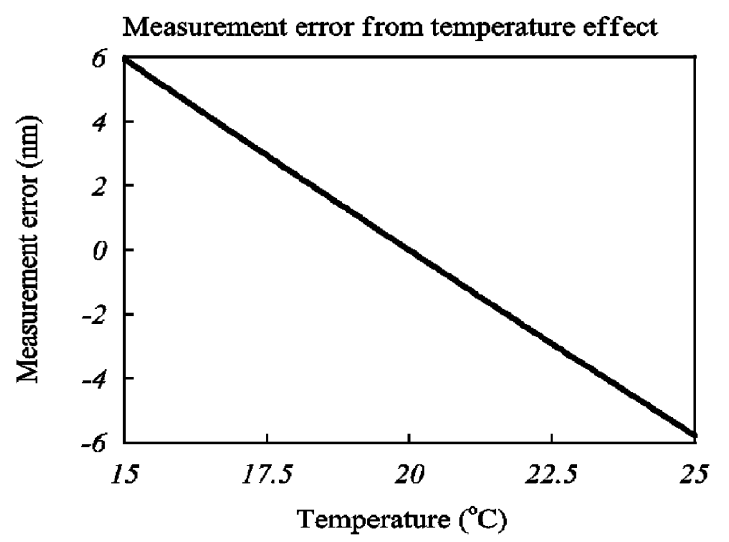

Fig. 5 The optical path difference induced by the difference between the operating temperature and the reference temperature $20^{\circ} \mathrm{C}$

and

$$
\varepsilon(\eta+\pi)=\varepsilon(\eta)
$$

By Eq. (13), it is clear that the polarization misalignment at the detector modules does induce an error with a period $\pi$. Since this error is periodic, it can improve the resolution of the system down to near $1 \mathrm{pm}$ for the corresponding quadrature signal processing by using the periodic average technique (Gürsel, 1993). Even for the additional phase difference of one degree, which is equivalent to an angle difference of $0.5^{\circ}$ stemming from the polarization misalignment of the detector module, there will exist a displacement measurement error larger than $1 \mathrm{~nm}$ which will make the measurement fail as shown clearly in Fig. 6.

\section{The Head-to-Scale Mechanical Runout}

There are five key degrees of freedom for the local grating scale relative to the optical head as shown in Fig. 1. The 3-D diffraction theory of the grating scale, i.e., the conical diffraction, is used to analyze the high-speed head-to-scale mechanical runout problem and leads to (Petit, 1980)

$$
\begin{aligned}
& u_{x n}=\sin \theta \cos \phi+\frac{n \lambda}{d}, \\
& u_{y n}=\sqrt{1-\sin ^{2} \phi-(\sin \theta \cos \phi+n \lambda / d)^{2}}, \\
& u_{z n}=\sin \phi,
\end{aligned}
$$

where $u_{x n}, u_{y n}$ and $u_{z n}$ are the $x$-, $y$ - and $z$-directional cosines of the diffracted beams respectively, $\phi$ is the inclined angle between the incident light beam and its projection light beam vector in the $x-y$ plane, and

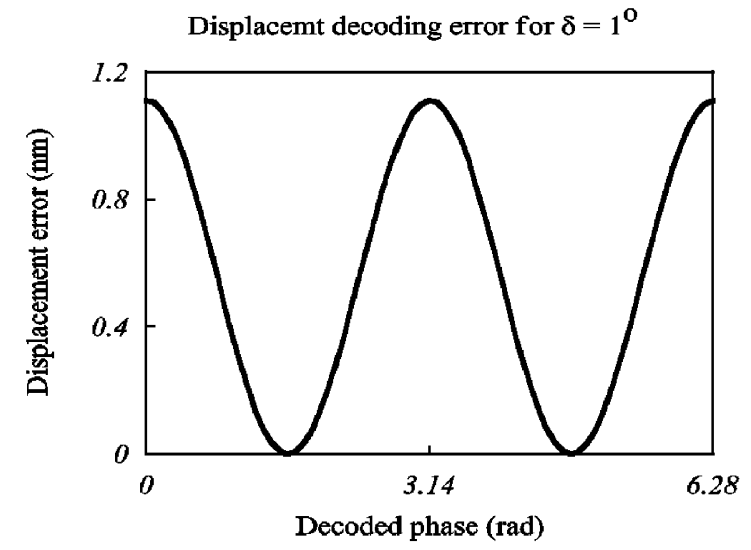

Fig. 6 Displacement error induced by the polarization misalignment of the detector module.

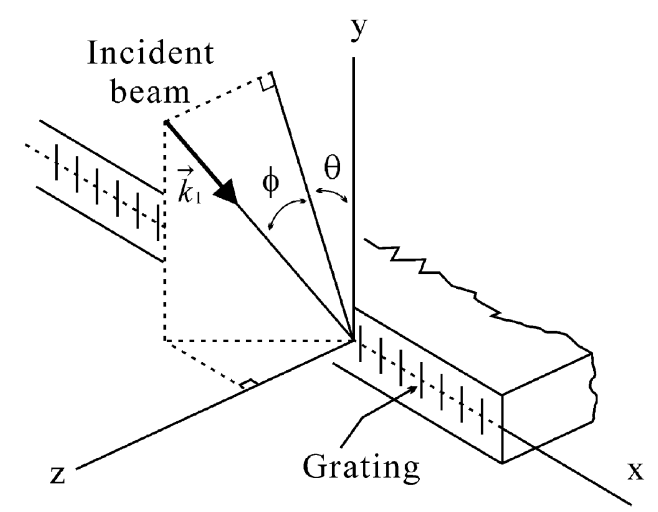

Fig. 7 The coordinate system used to examine conical diffraction

$\theta$ is the angle between the incident wave vector and the y-axis as shown in Fig. 7. With Eqs. (4) and (14)(16), $\alpha_{0}$ of the beam incident into the 1- $x$ telescope and its position of output beams onto the grating $h$ can be determined easily. For optical components 3 $\mathrm{mm}$ in size, the head-to-scale alignment tolerances are shown in Table 1 using LightTools ${ }^{\mathrm{TM}}$ raytrace calculations (Hayford et al., 1997; Canon Catalog, 1996).

For the system shown in Fig. 1, the roll and yaw are the runout components that are most difficult to minimize due to the nature of the stage movement. That is, the high-speed motion of the stage naturally leads to high roll and yaw runout. It is clear from the data shown in Table 1 that the newly developed laser encoder improves the most critical runout by a factor of 6 to 20. This significant improvement leads to a laser encoder that is much easier to implement and has a much higher performance.

\section{OPTICAL SIMULATIONS AND EXPERIMENTAL RESULTS}

The main difference between the configuration 
Table 1 Comparison of the Head-to-scale alignment tolerances of the proposed encoder and the Canon design

\begin{tabular}{ccc}
\hline Alignment tolerance & The proposed encoder & Canon encoder \\
\hline Roll & \pm 60 arc min & \pm 3 arc min \\
Pitch & \pm 20 arc min & \pm 20 arc min \\
Yaw & \pm 3 degrees & \pm 20 arc min \\
Stand-off & $\pm 1.2 \mathrm{~mm}$ & $\pm 0.2 \mathrm{~mm}$ \\
Offset & $\pm 2 \mathrm{~mm}$ & $\pm 0.3 \mathrm{~mm}$ \\
\hline
\end{tabular}

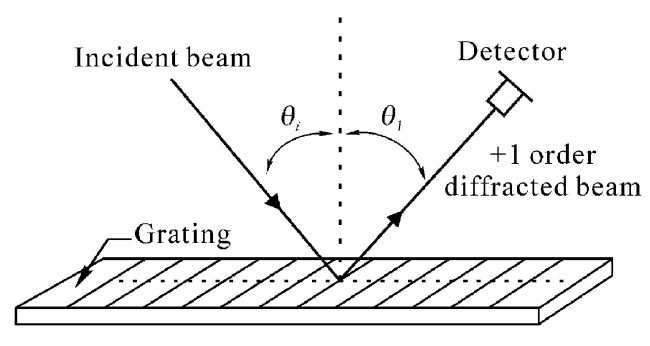

Fig. 8 The schematic of experimental setup used to measure the diffraction efficiency for the first order diffracted light beams.

of the present laser encoder and the IBM laser encoder design is that one instead of two $1-x$ telescopes is needed in the laser encoder configuration (Chiang and Lee, 1995; Hsieh and Lee, 1998; Lee and Hsieh, 1998).

Figure 8 shows the experimental setup used to measure diffraction efficiency. The linear grating used has a pitch of $1.6 \mu \mathrm{m}$, and is a sinusoidal surface relief type. In addition, the depth of the grating is $150 \mathrm{~nm}$ and the light source wavelength used is $632.8 \mathrm{~nm}$. Fig. 9 shows the measured and the calculated results. Fig. 10 represents a good agreement regardless of TE/TM waves or the Wood's anomaly between results by the GSolver ${ }^{\mathrm{TM}}$ program calculation and the published experimental data respectively. Note that solid lines are TM waves and dash lines are TE ones. It is clear from Figs. 9 and 10 that there exists good agreement between the results simulated by using the rigorous coupled wave theory and experimental results (Grating Solver Development Co., 1991). The design point of the grating scale lies on the $\left[k_{p}(\theta) k_{p}(0)+k_{s}(\theta) k_{s}(0)\right]^{2}$ that is proportional to the $A C$ component amplitude of the quadrature signal. During the course of developing the laser encoder presented in this article, the rigorous coupled wave theory was used to determine the optimum grating depth in order to lead to higher diffraction efficiency and a better $S / N$ ratio. Fig. 11 shows the proposed design point comparison of the grating scale with Canon's encoder. It is clear that the proposed design point, $190 \mathrm{~nm}$, will give more

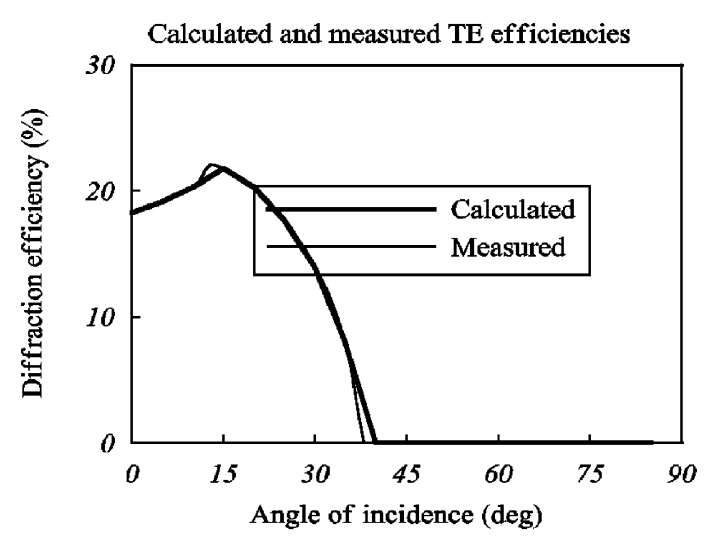

(a)

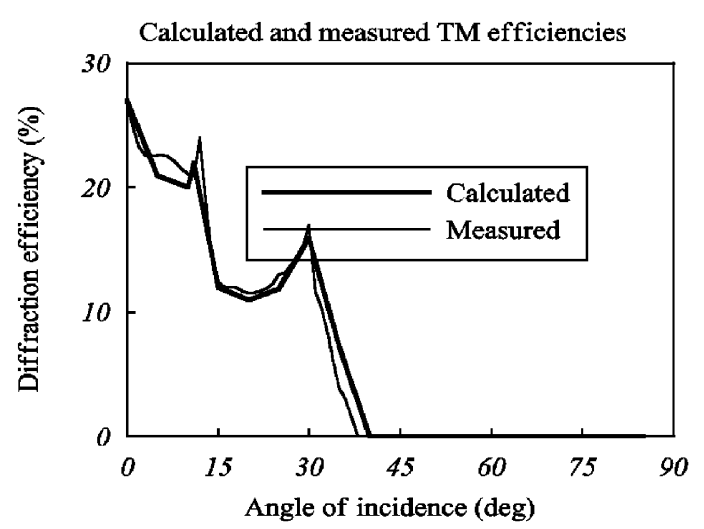

(b)

Fig. 9 The diffraction efficiency at various incident angles for a case where the grating scale is of a sinusoidal surface relief type, $1.6 \mu \mathrm{m}$ pitch, and $150 \mathrm{~nm}$ grating depth with gold coating: (a) and (b) show the calculated and measured diffraction efficiencies for TE and TM waves respectively.

than four times the relative signal amplitude than the Canon' design.

There is no suitable instrument which can be used for subnanometer resolution calibration. To further verify the system performance of the newly developed laser encoder system, its performance was compared with $\mathrm{HP}^{\mathrm{TM}}$ 5529A distance measuring interferometer (Fig. 12), i.e., same stage movement was measured by using both the newly developed laser 


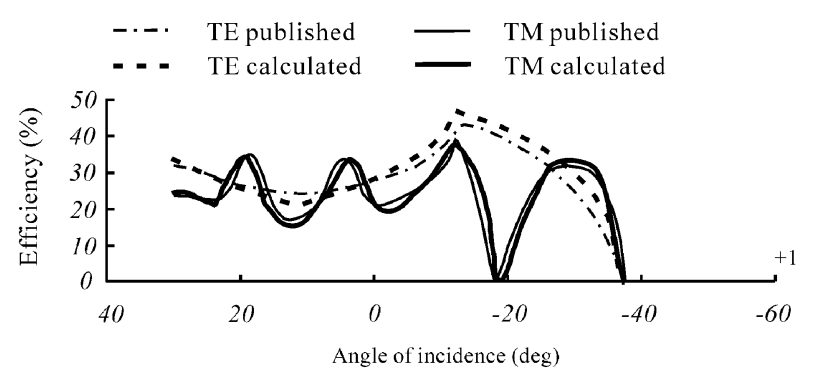

(a)

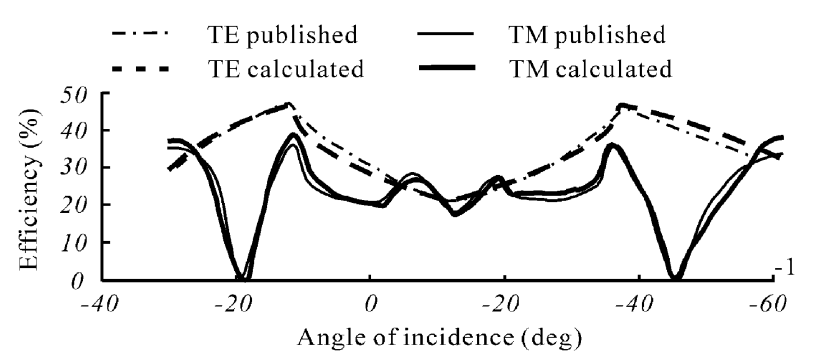

(b)

Fig. 10 The efficiencies as functions of angles of incidence in \pm 1 orders for a 730 lines per millimeter sinusoidal grating with depth of $200 \mathrm{~nm}$ : (a) +1 order; (b) -1 order. (The published experimental results are from Hutley and Bird, 1973)

encoder and $\mathrm{HP}^{\mathrm{TM}}$ 5529A. The experimental data obtained is shown in Fig. 13, which clearly demonstrates the performance of this newly developed laser encoder. The discrepancy between the signals obtained from the laser encoder and the HP interferometer can be traced to the oscillations of the mounting fixtures that hold the optical components of the tabletop laser encoder presented. It is expected that the performance will be significantly improved either by enhancing the mounting fixtures or by setting up a miniature laser encoder prototype in the near future.

\section{CONCLUSIONS}

A linear laser encoder that adopts the $1-x$ telescope concept was developed to provide a solution to counter the errors induced by practical mechanical runout of the specimen. The optical principle and the design concept of this newly developed system were also examined. Influence of laser encoder performance by the grating characteristics including the incidence angle, the surface profile, the grating depth, etc. was also detailed. The error from grating diffraction efficiency discrepancies of the incident light beams of different polarization states was evaluated. The effect of temperature and the polarization misalignment of the photodetectors are very important for measurement accuracy and resolution. It was

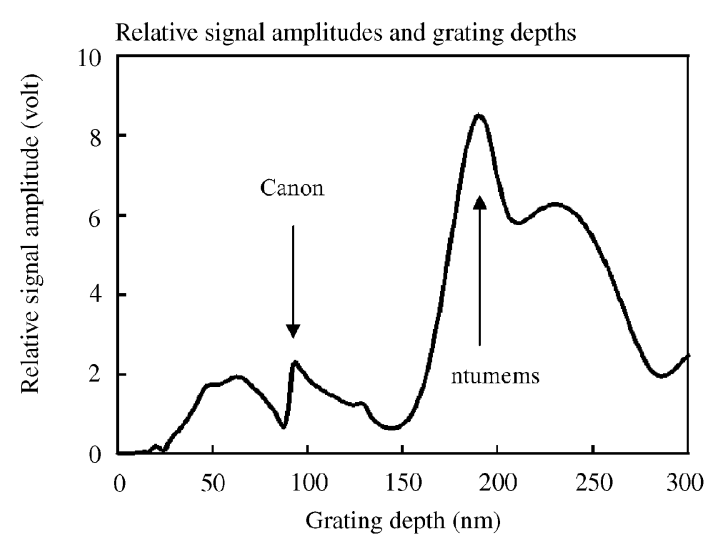

Fig. 11 The design point of the grating scale for the proposed encoder (shown as ntumems at near 190nm depth) can give more than four times the relative quadrature signal amplitude of the Canon's design

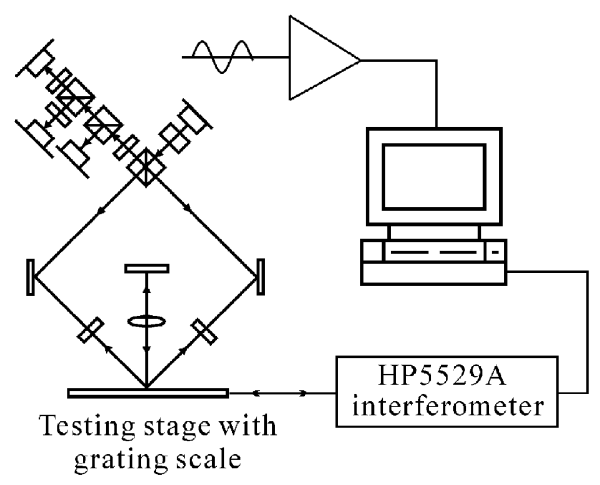

Fig. 12 The experimental setup for the performance test of the laser linear encoder

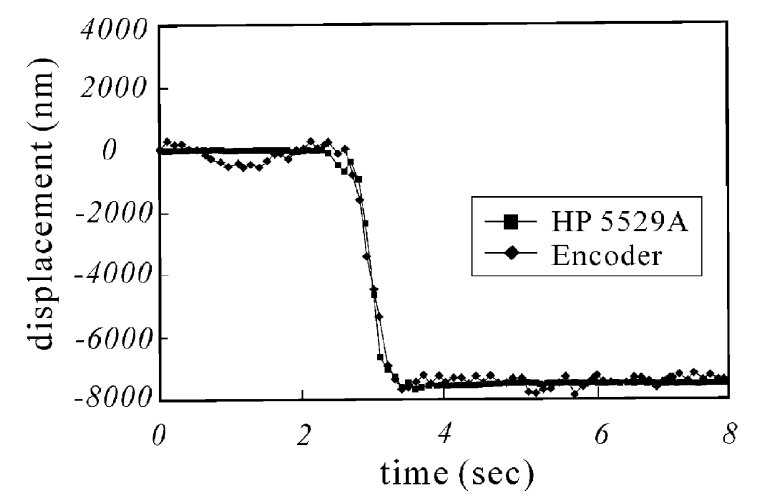

Fig. 13 Comparisons of the measured stage displacement between HP 5529A interferometer and the newly developed laser encoder

found that the grating scale should be viewed as a fundamental parameter of the optical design for linear laser encoders. The underlying reason that the newly developed laser encoder can have a much better head-to-scale alignment tolerance was also 
detailed. Experimental data obtained by using both a newly developed tabletop laser encoder and an HP distance measuring interferometer was found to be in good agreement.

\section{ACKNOWLEDGEMENTS}

The authors gratefully acknowledge the support of the National Science Council of Taiwan, R.O.C. under the Industrial University Collaborative project numbers: NSC 88- 2622-E-002-001, NSC 88-2622E-002-002, NSC 85-2622-E-002-017R, and NSC 862622- E-002-023R.

\section{NOMENCLATURE}

$a$

$\alpha_{0}$

$A_{1}$

$A_{2} \quad$ amplitude of the sinusoidal AC component of beam $2\left(\mathrm{~W} / \mathrm{m}^{2}\right)$

$b \quad$ sum of the multiplications of the diffraction efficiencies (dimensionless)

$d \quad$ the grating pitch (m)

$D$ transfer matrix for quarter waveplate when the light wave goes backward (dimensionless)

$D C_{1}$ amplitude of the DC component of beam 1 $\left(\mathrm{W} / \mathrm{m}^{2}\right)$

$D C_{2}$ amplitude of the DC component of beam 2 $\left(\mathrm{W} / \mathrm{m}^{2}\right)$

$d l \quad$ the infinitesimal optical path along path 1 and path2 (m)

$E_{1} \quad$ electric field (volt $\left./ \mathrm{m}\right)$

$E_{2} \quad$ electric field (volt $\left./ \mathrm{m}\right)$

$\boldsymbol{E}_{2}^{\prime}$ electric field (volt $\left./ \mathrm{m}\right)$

$G$ the proportional constant $\left(\mathrm{sec} / \mathrm{m}^{2}\right)$

$I_{1} \quad$ light intensity measured by PD1 $\left(\mathrm{W} / \mathrm{m}^{2}\right)$

$I_{2} \quad$ light intensity measured by $\mathrm{PD} 2\left(\mathrm{~W} / \mathrm{m}^{2}\right)$

$I_{2}^{\prime} \quad$ light intensity measured by PD2 $\left(\mathrm{W} / \mathrm{m}^{2}\right)$

$n$ the order of the diffraction (dimensionless)

$p \quad p$-polarization component of the wave (volt $/ \mathrm{m})$

$\boldsymbol{R}$ transfer matrix for quarter waveplate when the light wave goes forward (dimensionless)

$s \quad s$-polarization component of the wave (volt $/ \mathrm{m})$

$\boldsymbol{T}$ polarization transmission matrix (dimensionless)

$\boldsymbol{U}_{1} \quad$ polarization vector $(\mathrm{volt} / \mathrm{m})$

$\boldsymbol{U}_{2}$ polarization vector (volt $/ \mathrm{m}$ )

$u_{x n} \quad$ the direction cosine of the $n^{\text {th }}$ order diffracted beam with respect to the $\mathrm{x}$-axis (dimensionless)

$u_{y n} \quad$ the direction cosine of the $n^{\text {th }}$ order diffracted beam with respect to the $y$-axis (dimensionless)

$u_{z n} \quad$ the direction cosine of the $n^{\text {th }}$ order diffracted beam with respect to the $\mathrm{z}$-axis (dimensionless)

$\boldsymbol{V}_{1}$ polarization vector $(\mathrm{volt} / \mathrm{m})$

$V_{2}$ polarization vector $(\mathrm{volt} / \mathrm{m})$

$\boldsymbol{W}_{1}$ polarization vector $(\mathrm{volt} / \mathrm{m})$

$\boldsymbol{W}_{2}$ polarization vector (volt $/ \mathrm{m}$ )

$\Delta x \quad$ grating scale displacement (m)

\section{Greek Symbols}

$\beta \quad$ the designed incident angle of the encoder (rad)

$\delta \quad$ the additional phase difference between the two photo-detectors receiving the $I_{1}, I_{2}$ signals (rad)

$\Delta \omega$ the Doppler angular frequency shift $(1 / \mathrm{sec})$

$\phi \quad$ angle of incidence between the incident light beam vector and its projection on the $x-y$ plane (rad)

$\eta \quad$ relative phase difference ( $\mathrm{rad})$

$\gamma \quad$ phase lag (rad)

$\theta \quad$ angle of incidence in the incident plane (rad)

$\theta_{1}$ the diffracted angle of the first order of the diffracted beams (rad)

$\theta_{i} \quad$ the angle of incidence (rad)

$\theta_{m}$ the diffracted angle of the $m^{\text {th }}$ order of the diffracted beams (rad)

$\omega \quad$ angular frequency of the laser source $(1 / \mathrm{sec})$

$\xi \quad$ the polarization misalignment angle ( $\mathrm{rad}$ )

\section{REFERENCES}

1. Chiang, W. W., and Lee, C. K., 1995, "Wavefront Reconstruction Optics for Use in Disk Drive Position Measurement System," U.S. Patent No. $5,442,172$.

2. Canon Catalog, 1996, Encoders: Products and Capabilities, New York.

3. Drain, L. E., 1980, The Laser Doppler Technique, John Wiley \& Sons, New York.

4. Durst, F., Melling, A., and Whitelaw, J. H., 1976, Principles and Practice of Laser Doppler Anemometry, Academic Press, New York.

5. Guenther, R., 1990, Modern Optics, John Wiley \& Sons, New York.

6. Grating Solver Development Co., 1991, GSolver V2.0 User's Manual, Allen, TX.

7. Gürsel, Y., 1993, "Laser Metrology Gauges for OSI," SPIE Vol. 1947, pp. 188-197.

8. Hayford, M. J., Walker, C. T., Kruglyak, A., Brown, L.M., and Corn, E.T., 1997, LightTools User's Guide, Optical Research Associates, Pasadena, CA.

9. Hecht, E., 1998, Optics, Addison Wesley Longman, New York.

10. Hogert, E. N., Rebollo, M. A., and Gaggioli, N. G., 1991, "Alignment and/or Tilting Measurement by Means of Conical Diffraction Phenomena," Optics \& Laser Technology, Vol. 23, No. 6, pp. 
341-344.

11. Hsieh, C. T., and Lee, C. K., 1998, "Cylindrical Type Laser Encoders with High Tolerance to Grating Defects and Misalignment," ROC Patent No. 096,048.

12. Hsieh, C. T., 1997, "Theoretical Design and Experimental Implementation of Cylindrical Diffractive Optical Encoders (in Chinese)," Ph. D. Dissertation, Department of Mechanical Engineering, National Taiwan University, Taipei, Taiwan, R.O.C.

13. Hutley, M. C., 1982, Diffraction Gratings, Academic Press, New York.

14. Juergens, R. C. (Eds.), 1998, CODE V Reference Manual, Optical Research Associates, Inc., Pasadena, CA.

15. Lee, C. K., and Hsieh, C. T., 1998, "Diffractive Grating Linear Laser Encoders with High Tolerance to Grating Defects and Misalignment," ROC Patent No. 099,284.

16. Lee, C. K., Wu, G. Y., Teng, C. T., Wu, W. J., Lin, C. T., Hsiao, W. H., Shih, H. C., Wang, J. S., Lin, S. C., Lin, C. C., Lee, C. F., and Lin, Y. C., 1999, “A High Performance Doppler Interferometer for Advanced Optical Storage Systems," Jpn. J. Appl. Phys., Vol. 38, pp. 17301741.

17. Masreliez, K. G., 1992, "Position Detector and Method of Measuring Position," U.S. Patent No. 5,104,225.

18. Nishimura, T., Ishizuka, K., Ishii, S., and Tsukiji, M., 1990a, "Relief Diffraction Grating," U.S. Patent No. 5,021,649.

19. Nishimura, T., Tsukiji, M., Ishii, S., and Ishizuka, K., 1990b, "Encoder for Forming Interference Fringes by Re-Diffracted Lights from an Optical Type scale and Photoelectrically Converting the Interference Fringes to Thereby Direct the Displacement of the Scale," U.S. Patent No. 4,930, 895.

20. Olivier, P., and Francois, C., 1990, "Diffraction Photoelectric Displacement Measuring Device," U.S. Patent No. 4,938,595.

21. Petit, R. (Eds.), 1980, Electromagnetic Theory of Gratings, Springer-Verlag, New York.

22. Smith, W. J., 1990, Modern Optical Engineering, McGraw-Hill, New York.

23. Souji, I., Hideki, O., and Seiji, S., 1990, "Diffraction-Type Optical Encoder with Improved Detection Signal Insensitivity to Optical Grating Gap Variation," U.S. Patent No. 4,943,716.

24. Wu, C. C., Chen, Y. C., Lee, C. K., Hsieh, C. T., Wu, W. J., and Lu, S. S., 1999, "Design Verifications of a Linear Laser Encoder with High Head-to-Scale Tolerance," SPIE Vol. 3779, pp. 73-82.

\section{APPENDIX}

\section{Proof of Effect of Differences in Polarization Diffraction}

When $p$ - and $s$-waves go forward through quarter waveplates which have the same fast and slow axis directions in the polarization space, they do see optical elements with the same transfer matrix $\boldsymbol{D}$ from the physical point of view:

$$
\boldsymbol{D}=\left[\begin{array}{ll}
1 / \sqrt{2} & i / \sqrt{2} \\
i / \sqrt{2} & 1 / \sqrt{2}
\end{array}\right],
$$

where $i=\sqrt{-1}$. Note that for the return paths, the fast and slow axes certainly exchange and the transfer matrix must be changed to $\boldsymbol{R}$ :

$$
\boldsymbol{R}=\left[\begin{array}{cc}
1 / \sqrt{2} & i / \sqrt{2} \\
-i / \sqrt{2} & 1 / \sqrt{2}
\end{array}\right]
$$

After being diffracted by the grating scale, the output beams toward quarter waveplates which are located at the right half and left half of optical paths respectively, have polarization states, $\boldsymbol{V}_{1}$ and $\boldsymbol{V}_{2}$, respectively as follows:

$$
\boldsymbol{V}_{1}=\left\{\begin{array}{l}
k_{p}(\theta) k_{p}(0) \\
k_{s}(\theta) k_{s}(0) i
\end{array}\right\},
$$

and

$$
\boldsymbol{V}_{2}=\left\{\begin{array}{l}
k_{p}(\theta) k_{p}(0) \\
k_{s}(\theta) k_{s}(0) i
\end{array}\right\},
$$

where $k_{p}(\theta), k_{p}(0), k_{s}(\theta)$ and $k_{s}(0)$ are the first order diffraction efficiencies with respect to the $\theta$ and 0 incident angles for $p$ - and $s$-polarizations respectively. Thus we can calculate the polarization states of path 1 and path2, by the simple matrix operation before their hitting at PBS again as follows:

$$
\begin{aligned}
& \boldsymbol{U}_{1}=\boldsymbol{R} \boldsymbol{V}_{1}=\left\{\begin{array}{c}
b \\
-a i
\end{array}\right\}, \\
& \boldsymbol{U}_{2}=\boldsymbol{R} \boldsymbol{V}_{2}=\left\{\begin{array}{c}
a \\
-b i
\end{array}\right\},
\end{aligned}
$$

where $\boldsymbol{U}_{1}$ and $\boldsymbol{U}_{2}$ are the polarization vectors in path 1 and path 2 respectively and

$$
\begin{aligned}
& a=\frac{1}{\sqrt{2}}\left[k_{p}(\theta) k_{p}(0)+k_{s}(\theta) k_{s}(0)\right], \\
& b=\frac{1}{\sqrt{2}}\left[k_{p}(\theta) k_{p}(0)-k_{s}(\theta) k_{s}(0)\right] .
\end{aligned}
$$


After $\boldsymbol{U}_{1}$ and $\boldsymbol{U}_{2}$ enter PBS the second time, the polarization selection effect gives $s$-component of $\boldsymbol{U}_{1}$ and $p$-component of $\boldsymbol{U}_{2}$ as its outputs. Thus these two linear polarized waves which pass through the quarter waveplate within the circular polarization interferometer configuration have the following electric fields, $\boldsymbol{W}_{1}$ and $\boldsymbol{W}_{2}$

$$
\begin{aligned}
& \boldsymbol{W}_{1}=\boldsymbol{D} \boldsymbol{U}_{1} e^{i(\omega+2 \Delta \omega) t}=\left\{\begin{array}{c}
a \\
-a i
\end{array}\right\} e^{i(\omega+2 \Delta \omega) t}, \\
& \boldsymbol{W}_{2}=\boldsymbol{D} \boldsymbol{U}_{2} e^{i(\omega-2 \Delta \omega) t}=\left\{\begin{array}{l}
a \\
a i
\end{array}\right\} e^{i(\omega-2 \Delta \omega) t},
\end{aligned}
$$

where $\boldsymbol{W}_{1}$ and $\boldsymbol{W}_{2}$ are electric fields from path 1 and path 2 respectively, $\omega$ is the frequency of laser source, $\Delta \omega$ is the Doppler angular frequency shift, and $t$ is the propagation time of light wave. By Eqs. (A9) and (A10), we can find that both $\boldsymbol{W}_{1}$ and $\boldsymbol{W}_{2}$ are circularly polarized waves which have the same amplitude as the electric field. Note that the polarization transmission matrix $\boldsymbol{T}$ of a polarizer for a transmission angle, $\psi$, with respect to an axis parallel to the horizontal component of the corresponding electric field, can be represented as

$$
\boldsymbol{T}=\left[\begin{array}{cc}
\cos ^{2}(\psi) & \sin (\psi) \cos (\psi) \\
\sin (\psi) \cos (\psi) & \sin ^{2}(\psi)
\end{array}\right]
$$

For PD1 which couples with a polarizer which has a transmission direction at $\psi=0^{\circ}$, the received electric field $\boldsymbol{E}_{1}$, can be represented as

$$
\boldsymbol{E}_{1}=\left\{\begin{array}{c}
a e^{i(\omega+2 \Delta \omega) t}+a e^{i(\omega-2 \Delta \omega) t} \\
0
\end{array}\right\}
$$

Similarly, the electric field $\boldsymbol{E}_{2}$ which is received by PD2 with a polarizer of a transmission direction at $\psi=45^{\circ}$, can be represented as

$$
\boldsymbol{E}_{2}=\frac{1}{2}\left\{\begin{array}{l}
(a-a i) e^{i(\omega+2 \Delta \omega) t}+(a+a i) e^{i(\omega-2 \Delta \omega) t} \\
(a-a i) e^{i(\omega+2 \Delta \omega) t}+(a+a i) e^{i(\omega-2 \Delta \omega) t}
\end{array}\right\} .
$$

Therefore, the intensities $I_{1}$ and $I_{2}$ which are received by the PD1 and PD2, can be easily shown as follows

$$
\begin{aligned}
& I_{1}=2 a^{2}+2 a^{2} \cos (4 \Delta \omega \cdot t) . \\
& I_{2}=2 a^{2}+2 a^{2} \sin (4 \Delta \omega \cdot t) .
\end{aligned}
$$

Eqs. (A14) and (A15) show that the proposed optical design can avoid the effect of differences in the polarization diffraction efficiencies.

\section{Polarization Misalignment}

Now consider the polarization misalignment case in which there is a deviation angle at $\xi$, for the polarizer in front of the PD2. From Eqs. (A9) and (A10), we can assume two circularly polarized waves to transmit polarizers in front of PD1 and PD2. With the aid of Eq. (A11), the electric field $\boldsymbol{E}_{2}^{\prime}$ which is received by $\mathrm{PD} 2$, is as follows

$\boldsymbol{E}_{2}^{\prime}=a e^{i \omega}\left\{\begin{array}{l}(1-\sin (2 \xi) \cos (2 \Delta \omega \cdot t)+\cos (2 \xi) \sin (2 \Delta \omega \cdot t) \\ \cos (2 \xi)) \cos (2 \Delta \omega \cdot t)+(1+\sin (2 \xi) \sin (2 \Delta \omega \cdot t)\end{array}\right\}$.

(A16)

Thus, the signal $I_{2}^{\prime}$ which is received by PD2, can be represented as follows

$$
I_{2}=2 a^{2}+2 a^{2} \sin (4 \Delta \omega \cdot t+2 \xi)
$$

By Eq. (A17), we show the relation between the polarization misalignment and the phase deviation of the quadrature signal.

Manuscript Received: Jun. 12, 2000

Revision Received: Sep. 27, 2000 and Accepted: Oct. 12, 2000 


\title{
對機具高速運動偏擺具高對位公差之繞射式雷射 線性光學尺
}

\author{
吳乾埼 $^{1}$ 李世光 $^{2}$ 呂秀雄 $^{1}$ 陳文中 $^{1}$ 楊青桑 $^{2}$ 謝啓堂 $^{3}$ \\ 1 國立台灣大學機械工程學系 \\ 2 國立台灣大學應用力學研究所 \\ 3 華錦光電股份有限公司
}

\begin{abstract}
摘 要
本文提出一個具有高頭一尺對位公差的光學尺設計, 該設計採用單倍率望 遠鏡架構, 可使頭一尺間對位公差較同等級的 Canon 光學尺提高 6 至 20 倍。 本文所提光學設計，能避免光柵尺對 $p$ 線性偏極光與 $s$ 線性偏極光繞射效率的 差異的問題。頭一尺間些微的溫度場不均匀, 使正交訊號的初始相位不爲零, 降低光學尺系統的準確性。圓偏極光干涉儀架構中偏振板透振方向對位不準, 會產生斜隋圓正交信號，對於使用反正切解相位演算法的量測系統，其量測解 析度會降低。

關鍵詞：高速機具運動偏擺，誤差分析，單倍率望遠鏡，錐狀繞射。
\end{abstract}

